\title{
QUADRATIC TERM STRUCTURE MODELS FOR RISK-FREE AND DEFAULTABLE RATES
}

\author{
LI CHEN, DAMIR FILIPOVIĆ, AND H. VINCENT POOR
}

\begin{abstract}
In this paper, quadratic term structure models (QTSMs) are analyzed and characterized in a general Markovian setting. The primary motivation for this work is to find a useful extension of the traditional QTSM, which is based on an Ornstein-Uhlenbeck (OU) state process, while maintaining the analytical tractability of the model. To accomplish this, we introduce the class of quadratic processes, consisting of those Markov state processes which yield QTSMs. The main result states that OU processes are the only conservative quadratic processes. In general, however, a quadratic potential can be added to allow QTSMs to model default risk. It is further shown that the exponent functions that are inherent in the definition of the quadratic property can be determined by a system of Riccati equations with a unique admissible parameter set. The implications of these results for modeling the term structure of risk-free and defaultable rates are discussed.
\end{abstract}

\section{INTRODUCTION}

Recently, quadratic term structure models (QTSMs) have been studied in the context of both theoretical analysis and empirical testing (e.g., Ahn, Dittmar and Gallant 2002, Boyle and Tian 1999, Chen and Poor 2002, Cheng and Scaillet 2002, Gombani and Runggaldier 2001, Leippold and Wu (2002, 2003a, 2003b)). It has been shown that QTSMs not only empirically outperform the affine term structure models (ATSMs) in that they are able to capture the nonlinearity of the relevant time series and are more flexible for model design, but they also exhibit a nice analytical tractability comparable to ATSMs, namely, the zero-coupon bond price has an exponential-quadratic form in the state variables and the prices of European type options can be calculated by Fourier analysis. It is further shown in Filipović (2002) that the maximal degree of an arbitrage-free polynomial term structure model is two, which additionally underlines the importance of QTSMs.

The traditional QTSM is based on a $d$-dimensional Ornstein-Uhlenbeck (OU) state process, which, for every initial value $x \in \mathbb{R}^{d}$, can be realized as solution of

$$
d X_{t}=\left(\mu+\Lambda X_{t}\right) d t+\Sigma d W_{t}, \quad X_{0}=x,
$$

Date: November 2002 (first draft); September 23, 2003(this draft).

Key words and phrases. quadratic term structure models, option pricing, risk-free and defaultable rates, time-homogenous Markov processes. 
where $\mu \in \mathbb{R}^{d}, \Lambda, \Sigma \in \mathbb{R}^{d \times d}$ and $W$ is a $d$-dimensional standard Brownian motion on some filtered probability space (strictly speaking, (1.1) is a scaled and translated OU process, see e.g. Rogers and Williams 1994 [I.23]). The short rate is assumed to be a nonnegative quadratic function of $X$

$$
r\left(X_{t}\right)=R_{0}+\left\langle R_{1}, X_{t}\right\rangle+\left\langle R_{2} X_{t}, X_{t}\right\rangle \geq 0,
$$

for some appropriate $R_{0} \geq 0, R_{1} \in \mathbb{R}^{d}$ and $R_{2} \in \mathbb{R}^{d \times d}$. The QTSM (1.1)-(1.2) has the property that

$$
\begin{aligned}
& \mathbb{E}\left[e^{-\int_{t}^{T} r\left(X_{s}\right) d s} e^{\left\langle u, X_{T}\right\rangle+\left\langle V X_{T}, X_{T}\right\rangle} \mid \mathcal{F}_{t}\right] \\
& \quad=\exp \left(\tilde{A}(T-t, u, V)+\left\langle\tilde{B}(T-t, u, V), X_{t}\right\rangle+\left\langle\tilde{C}(T-t, u, V) X_{t}, X_{t}\right\rangle\right),
\end{aligned}
$$

for all $u \in \mathbb{C}^{d}$ and $V \in \mathbb{C}^{d \times d}$ such that $\exp (\langle u, x\rangle+\langle V x, x\rangle)$ is a bounded function. The deterministic functions $\tilde{A}, \tilde{B}$ and $\tilde{C}$ satisfy a system of Riccati equations with initial conditions

$$
\tilde{A}(0, u, V)=0, \quad \tilde{B}(0, u, V)=u \quad \text { and } \quad \tilde{C}(0, u, V)=V .
$$

This property turns out to be a crucial element for the analytical tractability of QTSMs: on setting $u=0$ and $V=0,(1.3)$ gives us price formulas for zero-coupon bonds. Moreover, as shown, e.g. in Leippold and $\mathrm{Wu}$ (2002), this property is the key to the Fourier analytic approach to pricing European options.

Several questions arise, which will be answered in this paper.

(1) Is it possible to add jumps to the state process (1.1) and retain (1.3)?

(2) What is the most general Markov process $X$ that satisfies (1.3)?

(3) How are the functions $\tilde{A}, \tilde{B}, \tilde{C}$ related to the generator of $X$ ?

The primary motivation is to find an extension of the traditional QTSM (1.1)-(1.2), while maintaining the analytical tractability of the model. However, it turns out that the answer to (1) is no. As for (2), we shall call $X$ "quadratic" if property (1.3) is satisfied. We give a complete characterization of quadratic processes within the class of "regular" (to be defined below) Markov processes with state space $\mathbb{R}^{d}$. It turns out that, if $X$ is conservative, then $X$ is necessarily an OU process (1.1). In general, $X$ can have a finite lifetime with a quadratic potential of the form (1.2). In either case, explicit expressions for (1.3) are available in terms of a system of Riccati equations, which replies to (3). This will prove useful when it comes to model default risk. Default risk can be incorporated in the present QTSMs in a way that is equivalent to the intensity-based credit risk modeling in the spirit of Jarrow, Lando and Turnbull (1997), Lando (1998) and Duffie and Singleton (1999). We discuss some particular QTSM pricing problems in detail.

In the case where the state space is a subset of $\mathbb{R}^{d}$, such as $\mathbb{R}_{+}^{m} \times \mathbb{R}^{n} \subset \mathbb{R}^{d}$, we provide examples which show that an extension of (1.1), e.g., including $X$ dependent diffusion or drift parts, while retaining (1.3) is difficult if not impossible. 
The remainder of this paper is organized as follows. In Section 2 we give the basic Markovian setup and introduce the notation that will be used in the text. Section 3 contains the definition of regular quadratic processes and our main characterization result. In Section 4 we give a general definition of a QTSM and link this to quadratic state processes. We derive bond (option) pricing formulas both for risk-free and defaultable interest rates. In particular, for the one-dimensional case $(d=1)$, closed-form expressions are available. In Section 5 we discuss some difficulties in extending quadratic processes to state spaces which differ from $\mathbb{R}^{d}$. A brief summary is given in Section 6. The Appendix contains some of the mathematical proofs.

\section{BASIC SETUP AND NOTATION}

For terminology and a general background on Markov processes we refer to Ethier and Kurtz (1986). We consider a contraction semigroup $\left(P_{t}\right)$ with transition function $p_{t}(x, d \xi)$, acting on $B\left(\mathbb{R}^{d}\right)$, the space of bounded and measurable functions $f: \mathbb{R}^{d} \rightarrow \mathbb{C}$, by

$$
P_{t} f(x)=\int_{\mathbb{R}^{d}} f(\xi) p_{t}(x, d \xi)
$$

We do not require that $\left(P_{t}\right)$ is conservative. If $p_{t}\left(x, \mathbb{R}^{d}\right)<1$ then we extend $p_{t}(x, \cdot)$ to $\overline{\mathbb{R}^{d}}=\mathbb{R}^{d} \cup\{\Delta\}$, the one-point compactification of $\mathbb{R}^{d}$, where $\Delta$ denotes the "point at infinity", by

$$
p_{t}(x,\{\Delta\})=1-p_{t}\left(x, \mathbb{R}^{d}\right), \quad p_{t}(\Delta,\{\Delta\})=1, \quad(t, x) \in \mathbb{R}_{+} \times \mathbb{R}^{d} .
$$

By convention, every function $f$ on $\mathbb{R}^{d}$ is extended to $\overline{\mathbb{R}^{d}}$ by setting

$$
f(\Delta):=0 \text {. }
$$

This holds in particular for the function $\mathbf{1}$, which is one on $\mathbb{R}^{d}$ and zero at $\Delta$.

We assume that $\left(P_{t}\right)$ can be realized on the space $\Omega$ of $\overline{\mathbb{R}^{d}}$-valued right continuous paths with left limits such that

$$
\omega(t)=\Delta \text { for } t>s \text { whenever } \omega(s-)=\Delta \text { or } \omega(s)=\Delta .
$$

We let $X_{t}(\omega)=\omega(t)$ denote the coordinate process, which induces the filtration $\mathcal{F}_{t}=\sigma\left(X_{s} \mid s \leq t\right)$ and $\sigma$-algebra $\mathcal{F}=\vee_{t \geq 0} \mathcal{F}_{t}$. Then $(\Omega, \mathcal{F})$ is equipped with the family of probability measures $\left\{\mathbb{P}_{x}\right\}_{x \in \mathbb{R}^{d}}$ such that $\mathbb{P}_{x}\left[X_{0}=x\right]=1$ and the Markov property

$$
\mathbb{E}_{x}\left[f\left(X_{t}\right) \mid \mathcal{F}_{s}\right]=\mathbb{E}_{X_{s}}\left[f\left(X_{t-s}\right)\right]=P_{t-s} f\left(X_{s}\right), \quad \mathbb{P}_{x} \text {-a.s. }
$$

holds for all $s \leq t$ and $x \in \mathbb{R}^{d}$, where $\mathbb{E}_{x}$ denotes expectation with respect to $x$. In view of (2.2), the stopping time (the lifetime of $X$ )

$$
T_{\Delta}:=\inf \left\{t \mid X_{t}=\Delta \text { or } X_{t-}=\Delta\right\}
$$


satisfies $X_{t}=\Delta$ for $t>T_{\Delta}$. We note that the path regularity of $X$ is not needed for the derivation of the characterization result in Section 3. In contrary, it is a consequence of Theorem 3.6. This assumption is solely made to simplify the discussion of QTSMs, which follows in Section 4, since there progressive measurability of $r(X)$ is needed. We shall write briefly $X$ for the Markov process $\left(X,\{\mathbb{P}\}_{x \in \mathbb{R}^{d}}\right)$ in what follows.

The Markov process $X$ and the semigroup $\left(P_{t}\right)$ are said to have the Feller property if

$$
P_{t} f \in C_{0}\left(\mathbb{R}^{d}\right) \quad \text { and } \quad \lim _{t \rightarrow 0} \sup _{x \in \mathbb{R}^{d}}\left|P_{t} f(x)-f(x)\right|=0 \quad \forall f \in C_{0}\left(\mathbb{R}^{d}\right),
$$

where $C_{0}\left(\mathbb{R}^{d}\right)$ denotes the space of continuous functions $f: \mathbb{R}^{d} \rightarrow \mathbb{C}$ which vanish at infinity.

We write $\mathrm{Sem}_{++}^{d}\left(\mathrm{Sem}_{+}^{d}\right)$ for the convex cone of symmetric positive (semi-) definite $d \times d$ matrices, respectively, and $\operatorname{Sem}_{-}^{d}:=-\operatorname{Sem}_{+}^{d}$ and $\operatorname{Sem}_{--}^{d}:=-\operatorname{Sem}_{++}^{d}$. Moreover,

$$
\langle\alpha, \beta\rangle:=\alpha_{1} \beta_{1}+\cdots+\alpha_{d} \beta_{d}, \quad \alpha, \beta \in \mathbb{C}^{d},
$$

and $e_{i}$ is the $i$-th standard orthonormal basis vector in $\mathbb{R}^{d}$. For two $d \times d$-matrices $A$ and $B$ we write $\operatorname{tr}(A B):=\sum_{i, j} A_{i j} B_{i j}$. The gradient and Hessian matrix of a function $f$ are denoted by $\nabla f$ and $\nabla^{2} f$, respectively. With $C_{c}^{2}\left(\mathbb{R}^{d}\right)$ we denote the space of twice continuously differentiable functions $f: \mathbb{R}^{d} \rightarrow \mathbb{C}$ with compact support in $\mathbb{R}^{d}$. All remaining notation is either standard or will be explained in the text.

\section{QUADRATIC PROCESSES}

In this section we define quadratic processes and provide the main characterization result. The convex cones

$$
\begin{aligned}
& \mathcal{E}:=\left\{(\gamma, \delta, \Phi) \in \mathbb{R} \times \mathbb{R}^{d} \times \operatorname{Sem}_{+}^{d} \mid \gamma+\langle\delta, x\rangle+\langle\Phi x, x\rangle \geq 0, \forall x \in \mathbb{R}^{d}\right\} \\
& \mathcal{B}:=\left\{(u, V) \in \mathbb{C}^{d} \times \mathbb{C}^{d \times d} \mid-\operatorname{Re}(\gamma, u, V) \in \mathcal{E} \text { for some } \gamma \in \mathbb{R}\right\}
\end{aligned}
$$

will be used frequently in this paper. It is clear that $(u, V) \in \mathcal{B}$ if and only if the continuous function

$$
f_{u, V}(x):=e^{\langle u, x\rangle+\langle V x, x\rangle}
$$

belongs to $B\left(\mathbb{R}^{d}\right)$.

Definition 3.1. The Markov process $X$ and the semigroup $\left(P_{t}\right)$ are called quadratic if $P_{t} f_{u, V}(x)$ has an exponential quadratic form in $x$, for every $(t, u, V) \in \mathbb{R}_{+} \times \mathcal{B}$. That is, there exist functions $A(t, u, V) \in \mathbb{C}, B(t, u, V) \in \mathbb{C}^{d}$ and $C(t, u, V) \in \mathbb{C}^{d \times d}$ such that

$$
P_{t} f_{u, V}(x)=\exp (A(t, u, V)+\langle B(t, u, V), x\rangle+\langle C(t, u, V) x, x\rangle)
$$


for every $(t, x, u, V) \in \mathbb{R}_{+} \times \mathbb{R}^{d} \times \mathcal{B}$.

Without loss of generality, since $\langle C x, x\rangle=\frac{1}{2}\left\langle\left(C+C^{\prime}\right) x, x\right\rangle$, we can and will assume that $C(t, u, V)$ is a symmetric matrix for any $(t, u, V) \in \mathbb{R}_{+} \times \mathcal{B}$.

Remark 3.2. Note that $B(t, u, V)$ and $C(t, u, V)$ are uniquely determined by (3.1), but $\operatorname{Im}(A(t, u, V))$ is determined only up to multiples of $2 \pi$. Therefore we make the convention that $A(t, \cdot, \cdot)$ denotes the unique continuous function on $\mathcal{B}$ with $A(t, 0,0)=0$

Remark 3.3. Since $P_{t} f_{u, V} \in B\left(\mathbb{R}^{d}\right)$, we have that $(B(t, u, V), C(t, u, V)) \in \mathcal{B}$, for all $(t, u, V) \in \mathbb{R}_{+} \times \mathcal{B}$. Since $P_{0} f=f$, we obtain the initial conditions

$$
A(0, u, V)=0, \quad B(0, u, V)=u \quad \text { and } \quad C(0, u, V)=V .
$$

Notice that $q \mapsto P_{t} f_{(i q, 0)}(x)$ is the characteristic function of the transition probability $p_{t}(x, \cdot)$, where $q \in \mathbb{R}^{d}$, and hence we see from (3.1) that the functions $A(t, u, V), B(t, u, V)$ and $C(t, u, V)$ uniquely determine the semigroup $\left(P_{t}\right)$. Definition 3.1 is an extension of the traditional QTSM-property (1.3), as shown by Corollary 3.8 and Proposition 4.4 below. The remaining task is to determine $A(t, u, V)$, $B(t, u, V)$ and $C(t, u, V)$ in terms of $\left(P_{t}\right)$. In order to do so we need further regularity and the notion of admissible parameters.

Definition 3.4. The Markov process $X$ and the semigroup $\left(P_{t}\right)$ are called regular if

(1) $\lim _{s \rightarrow t} p_{s}(x, \cdot)=p_{t}(x, \cdot)$ weakly on $\mathbb{R}^{d}$ (that is, $\lim _{s \rightarrow t} P_{s} f(x)=P_{t} f(x)$ for all $f \in C\left(\mathbb{R}^{d}\right) \cap B\left(\mathbb{R}^{d}\right)$ ) for all $(t, x) \in \mathbb{R}_{+} \times \mathbb{R}^{d} ;$ and

(2) the weak infinitesimal generator

$$
\tilde{\mathcal{A}} f_{u, V}(x):=\left.\partial_{t}^{+} P_{t} f_{u, V}(x)\right|_{t=0}=\lim _{t \downarrow 0} \frac{P_{t} f_{u, V}(x)-f_{u, V}(x)}{t}
$$

exists for every $(x, u, V) \in \mathbb{R}^{d} \times \mathcal{B}$, and $u \mapsto \tilde{\mathcal{A}} f_{u, 0}(x)$ is continuous at $u=0$, for all $x \in \mathbb{R}^{d}$.

Note that if $X$ is quadratic then condition (1) holds if and only if $A(t, u, V)$, $B(t, u, V)$ and $C(t, u, V)$ are continuous in $t \in \mathbb{R}_{+}$, for all $(u, V) \in \mathcal{B}$, by Lévy's continuity theorem for characteristic functions.

Definition 3.5. A parameter set $(\alpha, \beta, b, \gamma, \delta, \Phi)$ is said to be admissible if

$$
\alpha \in \operatorname{Sem}_{+}^{d}, \beta \in \mathbb{R}^{d}, b \in \mathbb{R}^{d \times d} \quad \text { and } \quad(\gamma, \delta, \Phi) \in \mathcal{E} .
$$

We are ready to formulate our main result, the proof of which is postponed to Appendix A.

Theorem 3.6. Suppose $X$ is a regular quadratic Markov process. Then it is a Feller process. Its infinitesimal generator $\mathcal{A}$ has $C_{c}^{2}\left(\mathbb{R}^{d}\right)$ as a core, and there exist 
admissible parameters $(\alpha, \beta, b, \gamma, \delta, \Phi)$ such that, for $f \in C_{c}^{2}\left(\mathbb{R}^{d}\right)$,

$$
\mathcal{A} f(x)=\operatorname{tr}\left(\alpha \nabla^{2} f(x)\right)+\langle\beta+b x, \nabla f(x)\rangle-(\gamma+\langle\delta, x\rangle+\langle\Phi x, x\rangle) f(x) .
$$

Moreover, the functions $A, B$ and $C$ in (3.1) satisfy the Riccati equations

$$
\begin{array}{lll}
\partial_{t} A(t, u, V)=F(B(t, u, V), C(t, u, V)), & A(0, u, V)=0, \\
\partial_{t} B(t, u, V)=R(B(t, u, V), C(t, u, V)), & B(0, u, V)=u, \\
\partial_{t} C(t, u, V)=T(B(t, u, V), C(t, u, V)), & C(0, u, V)=V,
\end{array}
$$

with

$$
\begin{aligned}
F(u, V) & =\langle\alpha u, u\rangle+2 \operatorname{tr}(\alpha V)+\langle\beta, u\rangle-\gamma, \\
R_{i}(u, V) & =4\left\langle\alpha u, V^{i}\right\rangle+\left\langle b_{i}, u\right\rangle+2\left\langle\beta, V^{i}\right\rangle-\delta_{i}, \\
T_{i j}(u, V) & =4\left\langle\alpha V^{i}, V^{j}\right\rangle+\left\langle b_{i}, V^{j}\right\rangle+\left\langle b_{j}, V^{i}\right\rangle-\Phi_{i j},
\end{aligned}
$$

where $V^{i}$ denotes the $i$-th column vector of $V$ and $b_{i}$ the $i$-th row vector of $b$.

Conversely, let $(\alpha, \beta, b, \gamma, \delta, \Phi)$ be admissible parameters. Then there exists a unique, regular quadratic semigroup $\left(P_{t}\right)$ with infinitesimal generator (3.3), and (3.1) holds where $A, B$ and $C$ are given as unique solutions of the Riccati equations (3.4)-(3.6).

We see from (3.3) that every trajectory of a regular quadratic process $X$ is continuous on $\left[0, T_{\Delta}\right.$ ) (see (2.3)), has a single jump to $\Delta$ at $T_{\Delta}$ and then remains in $\Delta$. If $X$ is conservative then $T_{\Delta}=+\infty, \mathbb{P}_{x}$-a.s., for all $x \in \mathbb{R}^{d}$. Non-conservative quadratic processes are useful for the modeling of defaultable rates, as is done in Section 4.2 below. It is convenient to have a criterion at hand to check whether $X$ is conservative or not. The following lemma is an immediate consequence of Theorem 3.6.

Lemma 3.7. Let $X$ be regular quadratic with parameters $(\alpha, \beta, b, \gamma, \delta, \Phi)$. Then the following are equivalent

(1) $X$ is conservative;

(2) $A(t, 0,0)=0, B(t, 0,0)=0$ and $C(t, 0,0)=0$ for all $t \geq 0$;

(3) $\gamma=0, \delta=0$ and $\Phi=0$.

It is easy to calculate the generator of the OU process (1.1). Theorem 3.6 now yields an immediate corollary.

Corollary 3.8. A conservative, regular quadratic process is an $O U$ process, and vice versa. The infinitesimal generator (3.3) and the stochastic differential equation (1.1) are related by

$$
\alpha=\Sigma \Sigma^{T} / 2, \quad \beta=\mu, \quad b=\Lambda .
$$


Cheng and Scaillet (2002) construct "quadratic" processes with non-trivial jump characteristics. These processes, however, satisfy (3.1) only for particular, $t$-dependent $V$, and not for all $V \in \operatorname{Sem}_{++}^{d}$ as here.

Remark 3.9. In what follows, the measures $\mathbb{P}_{x}$ will be considered as risk-neutral probabilities. It is, however, easy to perform an equivalent change of measure that preserves the quadratic structure of $X$. The reader is referred to Cheridito and Filipović (2003).

\section{QUADRATIC TERM STRUCTURE MODELS}

In this section, we give a general definition of quadratic term structure models, link them to quadratic state processes and discuss some pricing problems. The setup is as in Section 2. We fix $\left(R_{0}, R_{1}, R_{2}\right) \in \mathcal{E}$ and define the measurable function $r: \overline{\mathbb{R}^{d}} \rightarrow \mathbb{R}_{+}$by

$$
r(x):=R_{0}+\left\langle R_{1}, x\right\rangle+\left\langle R_{2} x, x\right\rangle .
$$

Note that in accordance with the convention (2.1) this means that $r(x)=0$ for $x=\Delta$. Recall that $X$ has right continuous paths with left limits, and hence is progressively measurable in particular, so that $\int_{0}^{t} r\left(X_{s}\right) d s$ is a well-defined $[0, \infty]$ valued, $\mathcal{F}_{t}$-measurable random variable. We can now define a new Markov semigroup.

Lemma 4.1. The linear operators $Q_{t}: B\left(\mathbb{R}^{d}\right) \rightarrow B\left(\mathbb{R}^{d}\right)$ given by

$$
Q_{t} f(x):=\mathbb{E}_{x}\left[e^{-\int_{0}^{t} r\left(X_{s}\right) d s} f\left(X_{t}\right)\right], \quad t \in \mathbb{R}_{+},
$$

form a Markov semigroup.

Proof. We clearly have $Q_{0} f=f$ and $0 \leq Q_{t} \mathbf{1} \leq 1$. Moreover, by the Markov property of $X$,

$$
\begin{aligned}
Q_{t+s} f(x) & =\mathbb{E}_{x}\left[e^{-\int_{0}^{t+s} r\left(X_{u}\right) d u} f\left(X_{t+s}\right)\right] \\
& =\mathbb{E}_{x}\left[e^{-\int_{0}^{t} r\left(X_{u}\right) d u} \mathbb{E}_{x}\left[\left(e^{-\int_{0}^{s} r\left(X_{u}\right) d u} f\left(X_{s}\right)\right) \circ \theta_{t} \mid \mathcal{F}_{t}\right]\right] \\
& =\mathbb{E}_{x}\left[e^{-\int_{0}^{t} r\left(X_{u}\right) d u} Q_{s} f\left(X_{t}\right)\right]=Q_{t} Q_{s} f(x), \quad \forall f \in B\left(\mathbb{R}^{d}\right),
\end{aligned}
$$

where $\theta_{t}(\omega)(s)=\omega(t+s)$ is the shift operator on $\Omega$. This yields the claim.

We shall interpret every $\mathbb{P}_{x}$ as risk-neutral measure. Then $Q_{t} f(x)$ is the price at time zero of the claim $f\left(X_{t}\right)$ due at $t$, given that the initial state $X_{0}$ is $x$. Henceforth we refer to $\left(Q_{t}\right)$ as the pricing semigroup.

Definition 4.2. We call $\left(X, R_{0}, R_{1}, R_{2}\right)$ a quadratic term structure model (QTSM) if the pricing semigroup $\left(Q_{t}\right)$ is regular quadratic and

$$
\mathbb{E}_{x}\left[e^{\int_{0}^{t} r\left(X_{s}\right) d s}\right]<\infty, \quad \forall(t, x) \in \mathbb{R}_{+} \times \mathbb{R}^{d} .
$$


Condition (4.3) asserts that the savings account is well behaved. It is satisfied if, roughly speaking, the eigenvalues of the mean-reversion rate matrix $b$ of $X$ are negative and large enough in absolute value. Condition 4.3 can be checked caseby-case in terms of some extended Riccati equations, as it is discussed in Duffie, Filipović and Schachermayer (2003) [Section 11.2]. For the one-dimensional case $(d=1)$, explicit solutions are given in Section 4.4 below. However, we do not go into further detail here.

Remark 4.3. The condition $\left(R_{0}, R_{1}, R_{2}\right) \in \mathcal{E}$ guarantees that the short rate $r\left(X_{t}\right)$ is nonnegative. It is a straightforward extension to replace $R_{0}$ by $R_{0}-c$, such that $r\left(X_{t}\right) \geq-c$, for some constant $c \in \mathbb{R}$.

That QTSMs are naturally linked to regular quadratic state processes $X$ is shown by the following version of the Feynman-Kac formula, the proof of which is postponed to Appendix B.

Proposition 4.4. The pricing semigroup $\left(Q_{t}\right)$ is regular quadratic with parameters $\left(\alpha, \beta, b, \gamma+R_{0}, \delta+R_{1}, \Phi+R_{2}\right)$ if and only if $\left(P_{t}\right)$ is regular quadratic with parameters $(\alpha, \beta, b, \gamma, \delta, \Phi)$.

Remark 4.5. For $d=1$, it is enough that the zero-coupon bond prices $Q_{t} \mathbf{1}(x)$ have an exponential-quadratic form in $x$ to conclude that $\left(P_{t}\right)$ is quadratic. See Filipovic (2001) for the affine case.

A QTSM $\left(X, R_{0}, R_{1}, R_{2}\right)$ with $R_{2} \in \operatorname{Sem}_{++}^{d}$ possesses a canonical representation, which is as follows. First, observe that (4.1) is equivalent to

$$
r(x)=\left\|R_{2}^{1 / 2}(x+c)\right\|^{2},
$$

where $R_{2}^{1 / 2}$ is the unique square root in $\operatorname{Sem}_{++}^{d}$ of $R_{2}, c$ is such that $R_{1}=2 R_{2} c$ and $R_{0}=\left\langle R_{2} c, c\right\rangle$ (there is some flexibility for $R_{0}$, see Remark 4.3). From Theorem 3.6 it is clear that $Y=R_{2}^{1 / 2}(X+c)$ is a regular quadratic process. Hence the QTSM $\left(X, R_{0}, R_{1}, R_{2}\right)$ is equal in law to the $\operatorname{QTSM}(Y, 0,0,1)$, where the short rates are given by

$$
r\left(Y_{t}\right)=\left\|Y_{t}\right\|^{2}
$$

Remark 4.6. QTSMs are not simply a subclass of affine term structure models in the sense of Duffie et al. (2003). Indeed, consider the one-dimensional OU process

$$
d Y_{t}=\left(\beta+b Y_{t}\right) d t+d W_{t},
$$

and let the short rates be of the canonical form $r_{t}=Y_{t}^{2}$ (see (4.4)). Using Itô's formula we derive

$$
d r_{t}=\left(2 \beta Y_{t}+2 b r_{t}+1\right) d t+2 \sqrt{r_{t}} d \tilde{W}_{t}
$$


with the Brownian motion $d \tilde{W}:=Y /|Y| d W$. Hence, neither $r$ nor $(r, Y)$ are affine processes in the sense of Duffie et al. (2003), where $(r, Y)$ would be allowed to take arbitrary initial values in $\mathbb{R}_{+} \times \mathbb{R}$.

"Quadratic term structure models" with $X$ following a Lévy process (meaning that the short rates are a quadratic function (4.1), which is not to be confused with our definition of a QTSM) have been investigated by Levendorskii (2002). There, however, the pricing semigroup is not quadratic in general and, consequently, there are no explicit formulae for bond prices such as (4.5) below. Instead, Levendorskii proposes an asymptotic approximation pricing strategy.

4.1. Bond Pricing. In order to price a zero-coupon bond with maturity $T$ we have to be aware that the valuated payoff is $\mathbf{1}\left(X_{T}\right)$, which can be less than one if $X$ is not conservative. This fact can be exploited to incorporate default risk into the QTSM, as is done in Section 4.2 below. In the absence of default risk we shall refer to the QTSM as "risk-free".

Definition 4.7. The $\operatorname{QTSM}\left(X, R_{0}, R_{1}, R_{2}\right)$ is called risk-free if the process $X$ is conservative (see Lemma 3.7).

In a risk-free QTSM we have $\mathbf{1}\left(X_{T}\right)=1$, so that the price $\pi(x, t, T)$ of a zerocoupon bond with maturity $T$ at time $t \leq T$, given that $X_{0}=x$, is of the form

$$
\begin{aligned}
\pi(x, t, T) & =\mathbb{E}_{x}\left[e^{-\int_{t}^{T} r\left(X_{s}\right) d s} \mid \mathcal{F}_{t}\right]=\mathbb{E}_{X_{t}}\left[e^{-\int_{0}^{T-t} r\left(X_{s}\right) d s} \mathbf{1}\left(X_{T}\right)\right] \\
& =Q_{T-t} \mathbf{1}\left(X_{t}\right)=e^{\tilde{A}(T-t, 0,0)+\left\langle\tilde{B}(T-t, 0,0), X_{t}\right\rangle+\left\langle\tilde{C}(T-t, 0,0) X_{t}, X_{t}\right\rangle},
\end{aligned}
$$

where $\tilde{A}, \tilde{B}$ and $\tilde{C}$ are the exponent functions which correspond to the regular quadratic semigroup $\left(Q_{t}\right)$.

4.2. Defaultable Rates. The fact that the quadratic process $X$ may be nonconservative allows us to incorporate default risk by letting the lifetime (2.3) model the default time. Since $\Delta$ is an absorbing state and by the convention (2.1), we can write consistently

$$
1_{\left\{T<T_{\Delta}\right\}}=1_{\left\{X_{T} \neq \Delta\right\}}=\mathbf{1}\left(X_{T}\right)
$$

which is the payoff at maturity $T$ of a defaultable zero-coupon bond with zero recovery. Suppose that $X$ is not conservative, that is, $(\gamma, \delta, \Phi) \neq(0,0,0)$, by Lemma 3.7. Then (4.5) represents the price of this defaultable zero-coupon bond.

Moreover, the risk-neutral probability that default occurs by time $T$, given that $X_{0}=x$, is

$$
\begin{aligned}
\mathbb{P}_{x}\left[T_{\Delta} \leq T\right] & =1-\mathbb{E}_{x}\left[1_{\left\{X_{T} \neq \Delta\right\}}\right]=1-P_{T} \mathbf{1}(x) \\
& =1-\exp \{A(T, 0,0)+\langle B(T, 0,0), x\rangle+\langle C(T, 0,0) x, x\rangle\} .
\end{aligned}
$$

Real-world default probabilities are easily obtained in a consistent way; see Remark 3.9 . 
The present QTSM can be considered as an intensity based default risk model in the spirit of Jarrow, Lando and Turnbull (1997) and Lando (1998). Indeed, let $\left(P_{t}^{0}, X, \mathbb{P}_{x}^{0}\right)$ denote the conservative regular quadratic process with parameters $(\alpha, \beta, b, 0,0,0)$. It induces a risk-free QTSM. We then define the default time (on an enlarged probability space)

$$
T_{d}^{0}:=\inf \left\{t \mid \int_{0}^{t} \lambda\left(X_{s}\right) d s \geq \phi\right\}
$$

where $\phi$ is a standard exponential random variable which is independent of $\mathcal{F}$, and

$$
\lambda(X):=\gamma+\langle\delta, X\rangle+\langle\Phi X, X\rangle
$$

models the intensity of default. By Proposition 4.4, the time zero price (4.5) equals

$$
\mathbb{E}_{x}^{0}\left[e^{-\int_{0}^{T} r\left(X_{s}\right) d s} e^{-\int_{0}^{T} \lambda\left(X_{s}\right) d s} \mathbf{1}\left(X_{T}\right)\right]
$$

which is the same as

$$
\mathbb{E}_{x}^{0}\left[e^{-\int_{0}^{T} r\left(X_{s}\right) d s} \mathbb{E}_{x}^{0}\left[\int_{0}^{T} \lambda\left(X_{s}\right) d s<\phi \mid \mathcal{F}\right]\right]=\mathbb{E}_{x}^{0}\left[e^{-\int_{0}^{T} r\left(X_{s}\right) d s} 1_{\left\{T<T_{d}^{0}\right\}}\right] .
$$

4.3. Option Pricing. Prices of bond options, both risk-free and defaultable, can now easily be obtained by taking advantage of the quadratic pricing semigroup. Consider a European put option on a zero-coupon bond with maturity $T$, strike price $K>0$ and exercise date $t<T$. The payoff at $t$ is $h(x, t, T)=(K-\pi(x, t, T))^{+}$, which is a continuous and bounded function of $x$, where $\pi(x, t, T)$ is given by (4.5). The price of this option at time 0 is

$$
\begin{aligned}
Q_{t} h(x, t, T)= & \mathbb{E}_{x}\left[e^{-\int_{0}^{t} r\left(X_{s}\right) d s} h(x, t, T)\right] \\
= & K \mathbb{E}_{x}\left[e^{-\int_{0}^{t} r\left(X_{s}\right) d s} 1_{\{\pi(x, t, T) \leq K\}}\right] \\
& -\mathbb{E}_{x}\left[e^{-\int_{0}^{t} r\left(X_{s}\right) d s} \pi(x, t, T) 1_{\{\pi(x, t, T) \leq K\}}\right] .
\end{aligned}
$$

We now define

$$
G_{u_{1}, V_{1} ; u_{2}, V_{2}}(y, x, t):=\mathbb{E}_{x}\left[e^{-\int_{0}^{t} r\left(X_{s}\right) d s} e^{\left\langle u_{1}, X_{t}\right\rangle+\left\langle V_{1} X_{t}, X_{t}\right\rangle} 1_{\left\{\left\langle u_{2}, X_{t}\right\rangle+\left\langle V_{2} X_{t}, X_{t}\right\rangle \leq y\right\}}\right],
$$

for $\left(u_{i}, V_{i}\right) \in \mathcal{B} \cap\left(R^{d} \times \mathbb{R}^{d \times d}\right)$. Then, given (4.5), (4.7) and (4.8), we have

$$
\begin{aligned}
Q_{t} h(x, t, T)= & K G_{0,0 ; \tilde{B}(T-t, 0,0), \tilde{C}(T-t, 0,0)}\left(K^{\prime}, x, t\right) \\
& -e^{\tilde{A}(T-t, 0,0)} G_{\tilde{B}(T-t, 0,0), \tilde{C}(T-t, 0,0) ; \tilde{B}(T-t, 0,0), \tilde{C}(T-t, 0,0)}\left(K^{\prime}, x, t\right),
\end{aligned}
$$

where

$$
K^{\prime}:=\ln (K)-\tilde{A}(T-t, 0,0) .
$$

Thus, we see that finding an analytical solution for the function $G$ is sufficient for solving European option pricing problems. 
It is seen from (4.8) that $G_{u_{1}, V_{1} ; u_{2}, V_{2}}(y, x, t)$ is the cumulative distribution function of $\left\langle u_{2}, X_{t}\right\rangle+\left\langle V_{2} X_{t}, X_{t}\right\rangle$ under the measure $e^{-\int_{0}^{t} r\left(X_{s}\right) d s} e^{\left\langle u_{1}, X_{t}\right\rangle+\left\langle V_{1} X_{t}, X_{t}\right\rangle} \mathbb{P}_{x}$. Therefore we can determine the function $G$ by calculating its characteristic function

$$
\begin{aligned}
\hat{G}_{u_{1}, V_{1} ; u_{2}, V_{2}}(z, x, t)= & \int_{\mathbb{R}} e^{i z y} G_{u_{1}, V_{1} ; u_{2}, V_{2}}(d y, x, t) \\
= & \mathbb{E}_{x}\left[e^{-\int_{0}^{t} r\left(X_{s}\right) d s} e^{\left\langle u_{1}+i z u_{2}, X_{t}\right\rangle+\left\langle\left(V_{1}+i z V_{2}\right) X_{t}, X_{t}\right\rangle}\right] \\
= & e^{\tilde{A}\left(t, u_{1}+i z u_{2}, V_{1}+i z V_{2}\right)+\left\langle\tilde{B}\left(t, u_{1}+i z u_{2}, V_{1}+i z V_{2}\right), x\right\rangle} \\
& \times e^{\left\langle\tilde{C}\left(t, u_{1}+i z u_{2}, V_{1}+i z V_{2}\right) x, x\right\rangle}
\end{aligned}
$$

By applying the inverse Fourier transform, we can easily obtain the prices of European options on zero-coupon bonds. This technique was originally proposed by Heston (1993), generalized by Duffie, Pan and Singleton (2000) to affine jumpdiffusion models, and by Leippold and Wu (2002), Cheng and Scaillet (2002) and others to quadratic term structure models.

4.4. One-Dimensional Case. For $d=1$ there are closed-form solutions of the Riccati equations (3.5)-(3.6) available, which makes most of the preceding formulas more explicit. For convenience, we rewrite here (3.4)-(3.6) for $A=A(t, u, V)$, $B=B(t, u, V)$ and $C=C(t, u, V)$

$$
\begin{aligned}
& \partial_{t} A=\alpha B^{2}+2 \alpha C+\beta B-\gamma, \quad A(0, u, V)=0, \\
& \partial_{t} B=(4 \alpha C+b) B+2 \beta C-\delta, \quad B(0, u, V)=u, \\
& \partial_{t} C=4 \alpha C^{2}+2 b C-\Phi, \quad C(0, u, V)=V .
\end{aligned}
$$

The solutions, as can be checked by straightforward (but cumbersome) calculations, are

$$
\begin{aligned}
& A(t, u, V)=\int_{0}^{t}\left(\alpha B(s, u, V)^{2}+2 \alpha C(s, u, V)+\beta B(s, u, V)-\gamma\right) d s \\
& B(t, u, V)=\frac{L_{1}(t) u+L_{2}(t) V+L_{3}(t)}{L_{4}(t) V+L_{5}(t)} \\
& C(t, u, V)=\frac{L_{6}(t) V+L_{7}(t)}{L_{4}(t) V+L_{5}(t)}
\end{aligned}
$$


where

$$
\begin{aligned}
L_{1}(t):= & 2 \Gamma e^{\Gamma t / 2} \\
L_{2}(t):= & -\frac{\beta}{2 \alpha} L_{4}(t)+\frac{16 \alpha}{\Gamma}\left(\frac{\beta b}{2 \alpha}+\delta\right)\left(e^{\Gamma t / 2}-1\right)^{2} \\
L_{3}(t):= & \frac{\beta}{2 \alpha}\left(2 \Gamma e^{\Gamma t / 2}-L_{5}(t)\right) \\
& -\left(\frac{\beta b}{2 \alpha}+\delta\right) \frac{4}{\Gamma}\left(e^{\Gamma t / 2}-1\right)\left(b\left(1-e^{\Gamma t / 2}\right)+\frac{\Gamma}{2}\left(1+e^{\Gamma t / 2}\right)\right), \\
L_{4}(t):= & 8 \alpha\left(1-e^{\Gamma t}\right) \\
L_{5}(t):= & \Gamma\left(e^{\Gamma t}+1\right)-2 b\left(e^{\Gamma t}-1\right) \\
L_{6}(t):= & \Gamma\left(e^{\Gamma t}+1\right)+2 b\left(e^{\Gamma t}-1\right) \\
L_{7}(t):= & 2 \Phi\left(1-e^{\Gamma t}\right)
\end{aligned}
$$

and $\Gamma:=2 \sqrt{b^{2}+4 \alpha \Phi}$.

For an efficient treatment of the Riccati equations (3.4)-(3.6) for $d \geq 2$, we refer the reader to Freiling (2002).

\section{Some Counter-Examples}

Given the rather restrictive result in Theorem 3.6 on the characteristics of regular quadratic processes with state space $\mathbb{R}^{d}$, one might try to generalize the setup and consider different state spaces, such as $\mathbb{R}_{+}^{m} \times \mathbb{R}^{n}$, which is the typical state space for affine processes, as in Duffie et al. (2003). However, this extension does not lead to more freedom in the choice of the characteristics of $X$ under the quadratic assumption, in general. The following two examples, for $m=n=1$, illustrate the difficulties.

5.1. Example 5.1. Consider the affine Markov process $X=(Y, Z)$ on $\mathbb{R}_{+} \times \mathbb{R}$ defined by

$$
\begin{aligned}
& Y_{t}=y \\
& Z_{t}=z+\int_{0}^{t} \sqrt{Y_{s}} d W_{s}=z+\sqrt{y} W_{t}
\end{aligned}
$$

with initial values $x=(y, z) \in \mathbb{R}_{+} \times \mathbb{R}$, where $W$ is a Brownian motion. This seems to be the simplest "stochastic volatility" process that is a quadratic candidate.

Let us check whether the quadratic property

$$
\mathbb{E}\left[e^{-w Z_{t}^{2}}\right]=e^{A(t, w)+\langle B(t, w), x\rangle+\langle C(t, w) x, x\rangle}
$$

can be satisfied for all $t, w \geq 0$ and $x \in \mathbb{R}_{+} \times \mathbb{R}$.

Straightforward calculations give

$$
e^{-w Z_{t}^{2}}=e^{-w z^{2}} e^{-2 w z \sqrt{y} W_{t}-w y W_{t}^{2}}=e^{-w z^{2}} e^{a U-U^{2}}
$$


where $a:=-2 \sqrt{w} z$ and $U:=\sqrt{w y} W_{t} \sim \mathcal{N}(0, \sigma)$ with $\sigma:=w y t$. Now

$$
\begin{aligned}
\mathbb{E}_{x}\left[e^{a U-U^{2}}\right] & =\frac{1}{\sqrt{2 \pi \sigma}} \int_{\mathbb{R}} e^{a s-s^{2}} e^{-\frac{s^{2}}{2 \sigma}} d s=\frac{1}{\sqrt{2 \pi \sigma}} \int_{\mathbb{R}} e^{-\frac{1}{2}\left((2+1 / \sigma) s^{2}-2 a s\right)} d s \\
& =\frac{1}{\sqrt{2 \pi \sigma}} \frac{1}{\sqrt{2+1 / \sigma}} e^{\frac{a^{2}}{4+2 / \sigma}} \int_{\mathbb{R}} e^{-\frac{1}{2}\left(t^{2}-2 \frac{a}{\sqrt{2+1 / \sigma}} t+\frac{a^{2}}{2+1 / \sigma}\right)} d t \\
& =\frac{1}{\sqrt{2 \sigma+1}} e^{\frac{a^{2}}{4+2 / \sigma}} .
\end{aligned}
$$

Hence the left hand side of (5.1) equals

$$
e^{-w z^{2}} \frac{1}{\sqrt{2 w y t+1}} e^{\frac{4 w z^{2}}{4+\frac{2}{w y t}}} .
$$

We see that (5.1) cannot hold, unless $w=0$.

5.2. Example 5.2. It is also impossible to allow for a simple "stochastic mean reversion level". Let $X=(Y, Z)$ be the affine Markov process on $\mathbb{R}_{+} \times \mathbb{R}$ defined by

$$
\begin{aligned}
& d Y_{t}=\sqrt{Y_{t}} d W_{t}, \\
& d Z_{t}=Y_{t} d t .
\end{aligned}
$$

Again, we have to check whether (5.1) can be satisfied. However, (5.1) and the Markov property of $X$ imply that, for fixed $T>0$ and $w \geq 0$,

$$
M_{t}:=\mathbb{E}\left[e^{-w Z_{T}^{2}} \mid \mathcal{F}_{t}^{X}\right]=e^{A(T-t, w)+\left\langle B(T-t, w), X_{t}\right\rangle+\left\langle C(T-t, w) X_{t}, X_{t}\right\rangle}, \quad t \in[0, T],
$$

is a martingale. Itô's formula yields

$$
\begin{gathered}
\frac{d M}{M}=\left(-A^{\prime}-\left\langle B^{\prime}, X\right\rangle-\left\langle C^{\prime} X, X\right\rangle+B_{2} Y+2 C_{21} Y^{2}+2 C_{22} Y Z\right. \\
\left.+\left(C_{11}+\frac{1}{2}\left(B_{1}+2 C_{11} Y+2 C_{12} Z\right)^{2}\right) Y\right) d t+\cdots d W
\end{gathered}
$$

The drift must be zero, which implies that the coefficients for all (mixed) powers of $X$ and $Y$ must vanish. For $Y^{3}$ this implies that $C_{11}=0$, and hence that $C_{12}=C_{21}=0$, by the semi-definiteness of $C$. But now the $Y Z$-term forces $C_{22}$ to be zero too. This is in conflict with the initial condition $C_{22}(0, w)=-w$, whence (5.1) cannot be satisfied, unless $w=0$.

5.3. Example 5.3. The above examples show that "square root" state variables cannot enter the dynamics of the "quadratic" state variables. However, it is generally possible for affine state variables to allow for a "quadratic mean reversion 
level". Let $X=(Y, Z)$ be the affine Markov process on $\mathbb{R}_{+} \times \mathbb{R}$ defined by

$$
\begin{aligned}
& d Y_{t}=Y_{t} d t+d W_{t}^{1}, \quad Y_{0}=y, \\
& d Z_{t}=\left(Y_{t}^{2}-Z_{t}\right) d t+\sqrt{Z_{t}} d W_{t}^{2}, \quad Z_{0}=z \geq 0,
\end{aligned}
$$

where, $W^{1}$ and $W^{2}$ are two independent Brownian motions. Then for any $u_{1} \in \mathbb{R}$ and $u_{2}, u_{3}<0$, it is straightforward to deduce that

$$
\mathbb{E}\left[e^{u_{1} Y_{T}+u_{2} Z_{T}+u_{3} Y_{T}^{2}}\right]=e^{A(T)+B_{y}(T) y+B_{z}(T) z+C(T) y^{2}},
$$

where

$$
\begin{aligned}
\partial_{t} A(t) & =C(t)+\frac{1}{2} B_{y}(t), \quad A(0)=0, \\
\partial_{t} B_{y}(t) & =2 B_{y}(t) C(t)+B_{y}(t), \quad B_{y}(0)=u_{1}, \\
\partial_{t} B_{z}(t) & =\frac{1}{2} B_{z}(t)^{2}-B_{z}(t), \quad B_{z}(0)=u_{2}, \\
\partial_{t} C(t) & =2\left(C(t)^{2}+C(t)\right)+B_{z}(t), \quad C(0)=u_{3} .
\end{aligned}
$$

\section{Conclusion}

In this paper, we have characterized the traditional quadratic term structure models (QTSMs) in a general Markovian setting, and demonstrated that in order to retain the analytical tractability, no jumps are allowed in the state processes of QTSMs. However, the state processes can incorporate a quadratic potential, which enables the QTSMs to model default risk. Moreover, we have shown that the pricing kernel is a quadratic semigroup so that the zero coupon bond pricing and option pricing formulas are straightforward to derive. Finally, we have explored the possibility of extending the quadratic processes into more general state spaces. The difficulty of this extension has been illustrated by the three simple examples given in Section 5 .

\section{Appendix A. Proof of Theorem 3.6}

We first provide some preliminary results, which will be used in the proof of Theorem 3.6. Throughout, $X$ denotes the Markov process with semigroup $\left(P_{t}\right)$ from Section 2 .

We start with a representation result for regular processes. Henceforth, we fix a uniformly bounded, continuous truncation function $\chi: \mathbb{R}^{d} \rightarrow \mathbb{R}^{d}$ with $\chi(\xi)=\xi$ on a neighborhood of 0 .

Lemma A.1. Suppose $\left(P_{t}\right)$ is a regular semigroup with weak infinitesimal generator $\tilde{\mathcal{A}}$. Then, for each $x \in \mathbb{R}^{d}$, there exist elements

$$
\alpha(x) \in \operatorname{Sem}_{+}^{d}, \quad \beta(x) \in \mathbb{R}^{d} \quad \text { and } \quad \gamma(x) \in \mathbb{R}_{+}
$$


and a nonnegative measure $\nu(x, d \xi)$ on $\mathbb{R}^{d} \backslash\{x\}$ satisfying

$$
\int_{\mathbb{R}^{d} \backslash\{x\}}\left(1 \wedge\|\xi-x\|^{2}\right) \nu(x, d \xi)<\infty,
$$

such that for all $(u, V) \in \mathcal{B}$

$$
\begin{aligned}
\tilde{\mathcal{A}} f_{u, V}(x)= & \operatorname{tr}\left(\alpha(x) \nabla^{2} f_{u, V}(x)\right)+\left\langle\beta(x), \nabla f_{u, V}(x)\right\rangle-\gamma(x) f_{u, V}(x) \\
& +\int_{\mathbb{R}^{d} \backslash\{x\}}\left(f_{u, V}(\xi)-f_{u, V}(x)-\left\langle\nabla f_{u, V}(x), \chi(\xi-x)\right\rangle\right) \nu(x, d \xi) .
\end{aligned}
$$

Proof. This lemma follows in the same way as Lemma 4.1 in Duffie et al. (2003).

Lemma A.2. Given $A \in \mathbb{R}^{d \times d}, \beta \in \mathbb{R}^{d}, \gamma \in \mathbb{R}$ and a measure $\nu$ on $\mathbb{R}^{d}$ with $\int_{\mathbb{R}^{d}}\left(1 \wedge\|\xi\|^{2}\right) \nu(d \xi)<\infty$, the representation

$$
\hat{\mu}(u)=\langle A u, u\rangle+\langle\beta, u\rangle+\gamma+\int_{\mathbb{R}^{d} \backslash\{0\}}\left[e^{\langle u, \xi\rangle}-1-\langle u, \chi(\xi)\rangle\right] \nu(d \xi), \quad u \in i \mathbb{R}^{d}
$$

is unique in terms of $A, \beta, \gamma$ and $\nu$.

Proof. See Theorem 8.1 in Sato (1999).

The following corollary is a direct extension of Lemma A.2.

Corollary A.3. Given $A, \beta, \gamma$ as in Lemma $A .2$ and $x \in \mathbb{R}^{d}$, the representation $\bar{\mu}(u, V)=\langle A(u+2 V x),(u+2 V x)\rangle+2 \operatorname{tr}(A V)+\langle\beta, u+2 V x\rangle-\gamma, \quad(u, V) \in \mathcal{B}$, is unique in terms of $A, \beta, \gamma$.

We introduce the set

$$
\Theta:=\left\{f_{u, V} \mid(u, V) \in \mathcal{B}, \operatorname{Re} V \in \operatorname{Sem}_{--}^{d}\right\}
$$

and its complex linear hull $\mathcal{L}(\Theta)$. It can be shown with similar arguments as in the proof of Lemma 8.4 in Duffie et al. (2003) that

$$
\mathcal{L}(\Theta) \text { lies dense in } C_{0}\left(\mathbb{R}^{d}\right) .
$$

Moreover, every $h \in C_{c}^{2}\left(\mathbb{R}^{d}\right)$ can be approximated by a sequence $\left(h_{k}\right)$ in $\mathcal{L}(\Theta)$ in the sense that

where we define the norm

$$
\lim _{k \rightarrow \infty}\left\|h_{k}-h\right\|_{\sharp}=0,
$$

$$
\|f\|_{\sharp}:=\sup _{x \in \mathbb{R}^{d}}\left\{(1+\|x\|)\left(|f(x)|+\|\nabla f(x)\|+\left\|\nabla^{2} f(x)\right\|\right)\right\} .
$$

After these preliminaries we are ready prove Theorem 3.6. 
Suppose first that $X$ is regular quadratic. Then the following right-hand derivatives exist

$$
\begin{aligned}
F(u, V) & :=\left.\partial_{t}^{+} A(t, u, V)\right|_{t=0}, \\
R(u, V) & :=\left.\partial_{t}^{+} B(t, u, V)\right|_{t=0}, \\
T(u, V) & :=\left.\partial_{t}^{+} C(t, u, V)\right|_{t=0},
\end{aligned}
$$

and thus we obtain

$$
\tilde{\mathcal{A}} f_{u, V}(x)=(F(u, V)+\langle R(u, V), x\rangle+\langle T(u, V) x, x\rangle) f_{u, V}(x),
$$

for all $(x, u, V) \in \mathbb{R}^{d} \times \mathcal{B}$. This in turn implies that

$$
\begin{aligned}
F(u, V) & =\tilde{\mathcal{A}} f_{u, V}(0), \\
R_{i}(u, V) & =\frac{1}{2}\left[\frac{\tilde{\mathcal{A}} f_{u, V}\left(e_{i}\right)}{f_{u, V}\left(e_{i}\right)}-\frac{\tilde{\mathcal{A}} f_{u, V}\left(-e_{i}\right)}{f_{u, V}\left(-e_{i}\right)}\right], \\
T_{i i}(u, V) & =\frac{1}{2}\left[\frac{\tilde{\mathcal{A}} f_{u, V}\left(e_{i}\right)}{f_{u, V}\left(e_{i}\right)}+\frac{\tilde{\mathcal{A}} f_{u, V}\left(-e_{i}\right)}{f_{u, V}\left(-e_{i}\right)}\right]-F(u, V),
\end{aligned}
$$

and

$$
\begin{aligned}
T_{i j}(u, V)= & \frac{1}{2} \frac{\tilde{\mathcal{A}} f_{u, V}\left(e_{i}+e_{j}\right)}{f_{u, V}\left(e_{i}+e_{j}\right)} \\
& -\frac{1}{2}\left[F(u, V)+R_{i}(u, V)+R_{j}(u, V)+T_{i i}(u, V)+T_{j j}(u, V)\right],
\end{aligned}
$$

for $i, j \in\{1,2, \ldots, d\}$ and $i \neq j$. Hence, we have to derive $\tilde{\mathcal{A}} f_{u, V}(x)$ for $x=s e_{i}+t e_{j}$, $s, t \in \mathbb{R}$, then $F, R$ and $T$ are determined. With the aid of Lemma A.2 this is indeed possible.

From (A.1) we have

$$
\begin{aligned}
\frac{\tilde{\mathcal{A}} f_{u, V}(x)}{f_{u, V}(x)}= & \langle\alpha(x)(u+2 V x), u+2 V x\rangle+2 \operatorname{tr}(\alpha(x) V)+\langle\beta(x), u+2 V x\rangle-\gamma(x) \\
& +\int_{\mathbb{R}^{d} \backslash\{x\}}\left(e^{\langle u, \xi-x\rangle+\langle V \xi, \xi\rangle-\langle V x, x\rangle}-1-\langle u+2 V x, \chi(\xi-x)\rangle\right) \nu(x, d \xi) \\
= & \langle\alpha(x)(u+2 V x), u+2 V x\rangle+2 \operatorname{tr}(\alpha(x) V)+\langle\beta(x), u+2 V x\rangle-\gamma(x) \\
& +\int_{\mathbb{R}^{d} \backslash\{0\}}\left(e^{\langle u+2 V x, \xi\rangle+\langle V \xi, \xi\rangle}-1-\langle u+2 V x, \chi(\xi)\rangle\right) \tilde{\nu}(x, d \xi)
\end{aligned}
$$

where $\tilde{\nu}(x, d \xi):=\nu(x, d(\xi+x))$. By applying (A.6) to (A.10), we obtain

$$
\begin{aligned}
F(u, V)= & \langle\alpha u, u\rangle+2 \operatorname{tr}(\alpha V)+\langle\beta, u\rangle-\gamma \\
& +\int_{\mathbb{R}^{d} \backslash\{0\}}\left(e^{\langle u, \xi\rangle+\langle V \xi, \xi\rangle}-1-\langle u, \chi(\xi)\rangle\right) m(d \xi),
\end{aligned}
$$


where

$$
\alpha:=\alpha(0), \beta:=\beta(0), \gamma:=\gamma(0) \text { and } m(d \xi):=\tilde{\nu}(0, d \xi) .
$$

In the same way, by applying (A.7) and (A.8) to (A.10), we derive that

$$
\begin{aligned}
R_{i}(u, V)= & \left\langle\hat{\alpha}_{i} u, u\right\rangle+4\left\langle\bar{\alpha}_{i} u+\hat{\alpha}_{i} V^{i}, V^{i}\right\rangle \\
& +2 \operatorname{tr}\left(\hat{\alpha}_{i} V\right)+\left\langle b_{i}, u\right\rangle+2\left\langle\bar{\beta}_{i}, V^{i}\right\rangle-\delta_{i} \\
& +\frac{1}{2} \int_{\mathbb{R}^{d} \backslash\{0\}}\left(e^{\left\langle u+2 V^{i}, \xi\right\rangle+\langle V \xi, \xi\rangle}-1-\left\langle u+2 V^{i}, \chi(\xi)\right) \nu_{i}(d \xi)\right. \\
& -\frac{1}{2} \int_{\mathbb{R}^{d} \backslash\{0\}}\left(e^{\left\langle u-2 V^{i}, \xi\right\rangle+\langle V \xi, \xi\rangle}-1-\left\langle u-2 V^{i}, \chi(\xi)\right) \nu_{-i}(d \xi),\right.
\end{aligned}
$$

where $V^{i}$ denotes the $i$-th column vector of $V$, and

$$
\begin{aligned}
T_{i i}(u, V)= & \left\langle\left(\bar{\alpha}_{i}-\alpha\right) u, u\right\rangle+4\left\langle\hat{\alpha}_{i} u+\bar{\alpha}_{i} V^{i}, V^{i}\right\rangle \\
& +2 \operatorname{tr}\left(\left(\bar{\alpha}_{i}-\alpha\right) V\right)+\left\langle\left(\bar{\beta}_{i}-\beta\right), u\right\rangle+2\left\langle b_{i}, V^{i}\right\rangle \\
& -\Phi_{i i}+\frac{1}{2} \int_{\mathbb{R}^{d} \backslash\{0\}}\left(e^{\left\langle u+2 V^{i}, \xi\right\rangle+\langle V \xi, \xi\rangle}-1-\left\langle u+2 V^{i}, \chi(\xi)\right) \nu_{i}(d \xi)\right. \\
& +\frac{1}{2} \int_{\mathbb{R}^{d} \backslash\{0\}}\left(e^{\left\langle u-2 V^{i}, \xi\right\rangle+\langle V \xi, \xi\rangle}-1-\left\langle u-2 V^{i}, \chi(\xi)\right) \nu_{-i}(d \xi)\right. \\
& -\int_{\mathbb{R}^{d} \backslash\{0\}}\left(e^{\langle u, \xi\rangle+\langle V \xi, \xi\rangle}-1-\langle u, \chi(\xi)\rangle\right) m(d \xi),
\end{aligned}
$$

for $1 \leq i \leq d$, where

$$
\begin{gathered}
\hat{\alpha}_{i}:=\frac{1}{2}\left(\alpha\left(e_{i}\right)-\alpha\left(-e_{i}\right)\right), \quad \bar{\alpha}_{i}:=\frac{1}{2}\left(\alpha\left(e_{i}\right)+\alpha\left(-e_{i}\right)\right), \\
b_{i}:=\frac{1}{2}\left(\beta\left(e_{i}\right)-\beta\left(-e_{i}\right)\right), \quad \bar{\beta}_{i}:=\frac{1}{2}\left(\beta\left(e_{i}\right)+\beta\left(-e_{i}\right)\right), \\
\delta_{i}:=\frac{1}{2}\left(\gamma\left(e_{i}\right)-\gamma\left(-e_{i}\right)\right), \quad \Phi_{i i}:=\frac{1}{2}\left(\gamma\left(e_{i}\right)+\gamma\left(-e_{i}\right)\right)-\gamma, \\
\nu_{i}(\cdot):=\tilde{\nu}\left(e_{i}, \cdot\right), \quad \text { and } \quad \nu_{-i}(\cdot):=\tilde{\nu}\left(-e_{i}, \cdot\right) .
\end{gathered}
$$

Now by (A.5), we have

$$
\frac{\tilde{\mathcal{A}} f_{u, V}\left(s e_{i}\right)}{f_{u, V}\left(s e_{i}\right)}=F(u, V)+s R_{i}(u, V)+s^{2} T_{i i}(u, V) .
$$

for all $s \in \mathbb{R},(u, V) \in \mathcal{B}$ and $i \in\{1,2, \ldots, d\}$.

Setting $V=0$ then, according to Lemma A.2 and given (A.10)-(A.14), we have

$$
\begin{aligned}
& \alpha\left(s e_{i}\right)=\alpha+s \hat{\alpha}_{i}+s^{2}\left(\bar{\alpha}_{i}-\alpha\right) \\
& \beta\left(s e_{i}\right)=\beta+s b_{i}+s^{2}\left(\bar{\beta}_{i}-\beta\right), \\
& \gamma\left(s e_{i}\right)=\gamma+s \delta_{i}+s^{2} \Phi_{i i},
\end{aligned}
$$


and

$$
\tilde{\nu}\left(s e_{i}, \cdot\right)=m(\cdot)+\frac{s}{2}\left(\nu_{i}(\cdot)-\nu_{-i}(\cdot)\right)+s^{2}\left(\frac{1}{2}\left[\nu_{i}(\cdot)+\nu_{-i}(\cdot)\right]-m(\cdot)\right) .
$$

Plugging this in, (A.14) now reads

$$
\begin{aligned}
0= & 4 s\left\langle\left(1-s^{2}\right)\left(\alpha-\bar{\alpha}_{i}\right) u, V^{i}\right\rangle+4\left(s^{2}-1\right)\left\langle\left(s^{2}\left(\bar{\alpha}_{i}-\alpha\right)+s \hat{\alpha}_{i}\right) V^{i}, V^{i}\right\rangle \\
& +2\left(s^{3}-s\right)\left\langle\left(\bar{\beta}_{i}-\beta\right), V^{i}\right\rangle \\
& +\int_{\mathbb{R}^{d} \backslash\{0\}}\left[e^{\langle V \xi, \xi\rangle+\left\langle u+2 s V^{i}, \xi\right\rangle}-1-\left\langle u+2 s V^{i}, \chi(\xi)\right\rangle\right] \tilde{\nu}\left(s e_{i}, d \xi\right) \\
& -\int_{\mathbb{R}^{d} \backslash\{0\}}\left[e^{\langle V \xi, \xi\rangle+\langle u, \xi\rangle}-1-\langle u, \chi(\xi)\rangle\right] m(d \xi) \\
& -\int_{\mathbb{R}^{d} \backslash\{0\}} \frac{s}{2}\left[e^{\langle V \xi, \xi\rangle+\left\langle u+2 V^{i}, \xi\right\rangle}-1-\left\langle u+2 V^{i}, \chi(\xi)\right\rangle\right] \nu_{i}(d \xi) \\
& +\int_{\mathbb{R}^{d} \backslash\{0\}} \frac{s}{2}\left[e^{\langle V \xi, \xi\rangle+\left\langle u-2 V^{i}, \xi\right\rangle}-1-\left\langle u-2 V^{i}, \chi(\xi)\right\rangle\right] \nu_{-i}(d \xi) \\
& -\int_{\mathbb{R}^{d} \backslash\{0\}} \frac{s^{2}}{2}\left[e^{\langle V \xi, \xi\rangle+\left\langle u+2 V^{i}, \xi\right\rangle}-1-\left\langle u+2 V^{i}, \chi(\xi)\right\rangle\right] \nu_{i}(d \xi) \\
& -\int_{\mathbb{R}^{d} \backslash\{0\}} \frac{s^{2}}{2}\left[e^{\langle V \xi, \xi\rangle+\left\langle u-2 V^{i}, \xi\right\rangle}-1-\left\langle u-2 V^{i}, \chi(\xi)\right\rangle\right] \nu_{-i}(d \xi) \\
& +\int_{\mathbb{R}^{d} \backslash\{0\}} s^{2}\left[e^{\langle V \xi, \xi\rangle+\langle u, \xi\rangle}-1-\langle u, \chi(\xi)\rangle\right] m(d \xi) .
\end{aligned}
$$

Now fix $V \in \operatorname{Sem}_{--}^{d}$ and let $u=i w$, for $w \in \mathbb{R}^{d}$. Write $I(w, \xi)$ for the integrand of the first integral in (A.19). Then

$$
\begin{aligned}
\partial_{w_{k}} I(w, \xi) & =i \xi_{k} e^{\langle V \xi, \xi\rangle+\left\langle i w+2 s V^{i}, \xi\right\rangle}-i \chi_{k}(\xi), \\
\partial_{w_{k}} \partial_{w_{l}} I(w, \xi) & =-\xi_{k} \xi_{l} e^{\langle V \xi, \xi\rangle+\left\langle i w+2 s V^{i}, \xi\right\rangle} .
\end{aligned}
$$

Hence

$$
\begin{aligned}
\left|\partial_{w_{k}} I(w, \xi)\right| & \leq C\left((1+\|w\|)\|\xi\|^{2} 1_{\left\{\xi \in B_{0}\right\}}+1_{\left\{\xi \notin B_{0}\right\}}\right), \\
\left|\partial_{w_{k}} \partial_{w_{l}} I(w, \xi)\right| & \leq C\left(\|\xi\|^{2} 1_{\left\{\xi \in B_{0}\right\}}+1_{\left\{\xi \notin B_{0}\right\}}\right),
\end{aligned}
$$

for some constant $C$ which does not depend on $w$, and where $B_{0}=\left\{\xi \mid\|\xi\|<\epsilon_{0}\right\}$ with $\epsilon_{0}>0$ is such that $\chi(\xi)=\xi$ on $B_{0}$. By dominated convergence it follows that we can differentiate twice with respect to $w$ under the integral signs in (A.19). 
Taking $\partial_{w_{1}}^{2}+\cdots+\partial_{w_{d}}^{2}$ in (A.19) yields

$$
\begin{aligned}
0= & \int_{\mathbb{R}^{d} \backslash\{0\}}\left[e^{i\langle w, \xi\rangle} e^{2 s\left\langle V^{i}, \xi\right\rangle}\|\xi\|^{2} e^{\langle V \xi, \xi\rangle}\right] \tilde{\nu}\left(s e_{i}, d \xi\right) \\
& -\int_{\mathbb{R}^{d} \backslash\{0\}} e^{i\langle w, \xi\rangle}\|\xi\|^{2} e^{\langle V \xi, \xi\rangle} m(d \xi) \\
& -\frac{s}{2} \int_{\mathbb{R}^{d} \backslash\{0\}} e^{i\langle w, \xi\rangle} e^{2\left\langle V^{i}, \xi\right\rangle}\|\xi\|^{2} e^{\langle V \xi, \xi\rangle} \nu_{i}(d \xi) \\
& +\frac{s}{2} \int_{\mathbb{R}^{d} \backslash\{0\}} e^{i\langle w, \xi\rangle} e^{-2\left\langle V^{i}, \xi\right\rangle}\|\xi\|^{2} e^{\langle V \xi, \xi\rangle} \nu_{-i}(d \xi) \\
& -\frac{s^{2}}{2} \int_{\mathbb{R}^{d} \backslash\{0\}} e^{i\langle w, \xi\rangle} e^{2\left\langle V^{i}, \xi\right\rangle}\|\xi\|^{2} e^{\langle V \xi, \xi\rangle} \nu_{i}(d \xi) \\
& -\frac{s^{2}}{2} \int_{\mathbb{R}^{d} \backslash\{0\}} e^{i\langle w, \xi\rangle} e^{-2\left\langle V^{i}, \xi\right\rangle}\|\xi\|^{2} e^{\langle V \xi, \xi\rangle} \nu_{-i}(d \xi) \\
& +s^{2} \int_{\mathbb{R}^{d} \backslash\{0\}} e^{i\langle w, \xi\rangle}\|\xi\|^{2} e^{\langle V \xi, \xi\rangle} m(d \xi) .
\end{aligned}
$$

Since this holds for all $w \in \mathbb{R}^{d}$ we obtain equality between the finite measures (see (A.18)),

$$
\begin{aligned}
e^{2 s\left\langle V^{i}, \xi\right\rangle}\|\xi\|^{2} e^{\langle V \xi, \xi\rangle}\left[m(d \xi)+\frac{s}{2}\left(\nu_{i}(d \xi)\right.\right. & \left.-\nu_{-i}(d \xi)\right) \\
& \left.+s^{2}\left(\frac{1}{2}\left[\nu_{i}(d \xi)+\nu_{-i}(d \xi)\right]-m(d \xi)\right)\right]
\end{aligned}
$$

and

$$
\begin{aligned}
\|\xi\|^{2} e^{\langle V \xi, \xi\rangle}[m(d \xi) & +\frac{s}{2}\left(e^{2\left\langle V^{i}, \xi\right\rangle} \nu_{i}(d \xi)-e^{-2\left\langle V^{i}, \xi\right\rangle} \nu_{-i}(d \xi)\right) \\
& \left.+s^{2}\left(\frac{1}{2}\left[e^{2\left\langle V^{i}, \xi\right\rangle} \nu_{i}(d \xi)+e^{-2\left\langle V^{i}, \xi\right\rangle} \nu_{-i}(d \xi)\right]-m(d \xi)\right)\right]
\end{aligned}
$$

for all $s \in \mathbb{R}$ and $V \in \operatorname{Sem}_{--}^{d}$. Now let $V=V(s)=\frac{W}{s}$ for some $W \in \operatorname{Sem}_{--}^{d}$, divide (A.21) and (A.22) by $s^{2}$ and let $s \rightarrow \infty$. Then we obtain, on any compact set in $\mathbb{R}^{d}$,

$$
\begin{aligned}
e^{2\left\langle W^{i}, \xi\right\rangle}\|\xi\|^{2}\left(\frac{1}{2}\left[\nu_{i}(d \xi)+\nu_{-i}(d \xi)\right]-\right. & m(d \xi)) \\
& =\|\xi\|^{2}\left(\frac{1}{2}\left[\nu_{i}(d \xi)+\nu_{-i}(d \xi)\right]-m(d \xi)\right) .
\end{aligned}
$$

Since this equality holds for any $W^{i}$ which is the $i$-th column vector of some $W \in$ Sem ${ }_{--}^{d}$, we conclude that

$$
\frac{1}{2}\left[\nu_{i}+\nu_{-i}\right]-m=0
$$


But this implies, since $\tilde{\nu}\left(s e_{i}, d \xi\right)$ in $($ A.18) is a nonnegative measure for all $s \in \mathbb{R}$, that

$$
\nu_{i}=\nu_{-i}=m=0 .
$$

Going back to (A.19) we now readily obtain, by Lemma A.2, that $\bar{\alpha}_{i}-\alpha=\hat{\alpha}_{i}=0$ and $\bar{\beta}_{i}-\beta=0$, whence

$$
\alpha\left(s e_{i}\right)=\alpha, \quad \beta\left(s e_{i}\right)=\beta+s b_{i} .
$$

We thus have proved (3.7), (3.8) and (3.9) for the diagonal elements.

As for the remaining part in (3.9), we consider the equation (see (A.5)),

$$
\begin{aligned}
& \frac{\tilde{\mathcal{A}} f_{u, V}\left(s e_{i}+t e_{j}\right)}{f_{u, V}\left(s e_{i}+t e_{j}\right)} \\
& =F(u, V)+s R_{i}(u, V)+t R_{j}(u, V)+s^{2} T_{i i}(u, V)+2 s t T_{i j}(u, V)+t^{2} T_{j j}(u, V),
\end{aligned}
$$

for all $s, t \in \mathbb{R}$. Combine this with (A.10) and the above results, set $V=0$ and apply Lemma A.2 to obtain

$$
\begin{aligned}
\alpha\left(s e_{i}+t e_{j}\right) & =\alpha, \\
\beta\left(s e_{i}+t e_{j}\right) & =\beta+s b_{i}+t b_{j}, \\
\gamma\left(s e_{i}+t e_{j}\right) & =\gamma+s \delta_{i}+t \delta_{j}+s^{2} \Phi_{i i}+2 s t \Phi_{i j}+t^{2} \Phi_{j j},
\end{aligned}
$$

where

and

$$
\Phi_{i j}:=\frac{1}{2}\left(\gamma\left(e_{i}+e_{j}\right)-\gamma-\delta_{i}-\delta_{j}-\Phi_{i i}-\Phi_{j j}\right)
$$

$$
\nu\left(s e_{i}+t e_{j}\right)=0 .
$$

This together with (A.9) completes the proof of (3.9).

From (A.5) we now obtain

$$
\begin{aligned}
\frac{\tilde{\mathcal{A}} f_{u, V}(x)}{f_{u, V}(x)}= & F(u, V)+\langle R(u, V), x\rangle+\langle T(u, V) x, x\rangle \\
= & \langle\alpha(u+2 V x), u+2 V x\rangle+2 \operatorname{tr}(\alpha V) \\
& +\langle\beta+b x, u+2 V x\rangle-(\gamma+\langle\delta, x\rangle+\langle\Phi x, x\rangle) .
\end{aligned}
$$

Lemma A.1 and Corollary A.3 imply that $\gamma+\langle\delta, x\rangle+\langle\Phi x, x\rangle$ must be nonnegative. Hence $(\gamma, \delta, \Phi) \in \mathcal{E}$, and $(\alpha, \beta, b, \gamma, \delta, \Phi)$ are admissible.

From the semigroup property $P_{t+s}=P_{s} P_{t}$ we obtain

$$
\begin{aligned}
& A(t+s, u, V)=A(t, u, V)+A(s, B(t, u, V), C(t, u, V)), \\
& B(t+s, u, V)=B(s, B(t, u, V), C(t, u, V)), \\
& C(t+s, u, V)=C(s, B(t, u, V), C(t, u, V)) .
\end{aligned}
$$


Thus the right-hand derivatives in $t$ of $A, B, C$ exist for all $t \geq 0$ and satisfy

$$
\begin{aligned}
& \partial_{t}^{+} A(t, u, V)=F(B(t, u, V), C(t, u, V)), \\
& \partial_{t}^{+} B(t, u, V)=R(B(t, u, V), C(t, u, V)), \\
& \partial_{t}^{+} C(t, u, V)=T(B(t, u, V), C(t, u, V)),
\end{aligned}
$$

for all $(u, V) \in \mathcal{B}$. But $A, B, C$ are continuous in $t$ by assumption and $F, R, T$ are smooth mappings. If follows that $A, B, C$ are continuously differentiable in $t$ and satisfy the Riccati equations (3.4)-(3.6).

For the Feller property of $X$ we observe that, since $C(t, u, V)$ is the solution of (3.6), $\operatorname{Re} C(t, u, V)$ lies in $\operatorname{Sem}_{--}^{d}$ if $\operatorname{Re} V \in \operatorname{Sem}_{--}^{d}$, for all $t \geq 0$. Thus $P_{t} f \in$ $C_{0}\left(\mathbb{R}^{d}\right)$ and $\lim _{t \rightarrow 0} \sup _{x \in \mathbb{R}^{d}}\left|P_{t} f(x)-f(x)\right|=0$ for all $f \in \mathcal{L}(\Theta)$, and hence for all $f \in C_{0}\left(\mathbb{R}^{d}\right)$, by (A.3). This implies that $\left(P_{t}\right)$ is a Feller semigroup.

Moreover, since $\left.\partial_{t}^{+} P_{t} f\right|_{t=0}=\tilde{\mathcal{A}} f \in C_{0}\left(\mathbb{R}^{d}\right)$, for all $f \in \mathcal{L}(\Theta)$, we infer by the Feller property (see Sato (1999) [Lemma 31.7]) that $\mathcal{L}(\Theta) \subset \mathcal{D}(\mathcal{A})$ and $\mathcal{A} f=\tilde{\mathcal{A}} f$ for all $f \in \mathcal{L}(\Theta)$. By the closedness of $\mathcal{A}$ and (A.4) it follows that $C_{c}^{2}\left(\mathbb{R}^{d}\right) \subset \mathcal{D}(\mathcal{A})$ and (3.3) holds for all $f \in C_{c}^{2}\left(\mathbb{R}^{d}\right)$.

As for cores, we observe that $P_{t} \mathcal{L}(\Theta) \subset \mathcal{L}(\Theta) \subset \mathcal{D}(\mathcal{A})$. From this it follows that $\mathcal{L}(\Theta)$ is a core of $\mathcal{A}$ (see Sato (1999) [Lemma 31.6]). Again, by the closedness of $\mathcal{A}$ and (A.4) we conclude that $C_{c}^{2}\left(\mathbb{R}^{d}\right)$ is a core of $\mathcal{A}$, and the first part of Theorem 3.6 is proved.

Conversely, let $(\alpha, \beta, b, \gamma, \delta, \Phi)$ be admissible parameters, and suppose first that $\Phi=0$. Then we are in the affine situation and Theorem 3.6 follows from the existence and uniqueness results for affine processes in Sato (1999).

For the general case, we let $X$ and $\left(P_{t}\right)$ be the regular quadratic process and semigroup corresponding to $(\alpha, \beta, b, \gamma, \delta, 0)$, and consider the semigroup $\left(Q_{t}\right)$ defined by (4.2) with $R_{0}=0, R_{1}=0$ and $R_{2}=\Phi$. From Proposition 4.4 it then follows that $\left(Q_{t}\right)$ is regular quadratic with parameters $(\alpha, \beta, b, \gamma, \delta, \Phi)$. The Feller property and uniqueness of $\left(Q_{t}\right)$ follows, as above, by (A.3) and since $\mathcal{L}(\Theta)$ is a core of the generator of $\left(Q_{t}\right)$. This completes the proof of Theorem 3.6.

\section{Appendix B. Proof of Proposition 4.4}

Let $\mathcal{A}: \mathcal{D}(\mathcal{A}) \subset B\left(\mathbb{R}^{d}\right) \rightarrow B\left(\mathbb{R}^{d}\right)$ and $\mathcal{G}: \mathcal{D}(\mathcal{G}) \subset B\left(\mathbb{R}^{d}\right) \rightarrow B\left(\mathbb{R}^{d}\right)$ denote the infinitesimal generators of $\left(P_{t}\right)$ and $\left(Q_{t}\right)$ on $B\left(\mathbb{R}^{d}\right)$ and

$$
R_{\lambda}^{P} g(x)=\int_{\mathbb{R}_{+}} e^{-\lambda t} P_{t} g(x) d t \quad \text { and } \quad R_{\lambda}^{Q} g(x)=\int_{\mathbb{R}_{+}} e^{-\lambda t} Q_{t} g(x) d t, \quad \lambda>0,
$$

their resolvents, respectively. They are well defined in either case. Also we define the closed linear operator $\mathcal{R}$ on $B\left(\mathbb{R}^{d}\right)$ with domain $\mathcal{D}(\mathcal{R})=\left\{f \in B\left(\mathbb{R}^{d}\right) \mid \mathcal{R} f \in\right.$ $\left.B\left(\mathbb{R}^{d}\right)\right\}$ by

$$
\mathcal{R} f(x):=r(x) f(x) .
$$


Suppose first that $\left(P_{t}\right)$ is a regular quadratic semigroup with parameters $(\alpha, \beta, b$, $\gamma, \delta, \Phi)$. Then it can be shown as in the proof of Proposition 11.1 in Duffie et al. (2003) (see also Rogers and Williams (1994) [Section III.19]) that

$$
R_{\lambda}^{P} g=R_{\lambda}^{Q}\left(g+\mathcal{R}\left(R_{\lambda}^{P} g\right)\right)
$$

for all $g \in C_{0}\left(\mathbb{R}^{d}\right)$ with $R_{\lambda}^{P} g \in \mathcal{D}(\mathcal{R})$. By the Feller property of $\left(P_{t}\right)$ it follows that $R_{\lambda}^{P}=(\lambda-\mathcal{A})^{-1}: C_{0}\left(\mathbb{R}^{d}\right) \rightarrow \mathcal{D}(\mathcal{A}) \cap C_{0}\left(\mathbb{R}^{d}\right)$ is a bijection, and from Theorem 3.6 we know that $C_{c}^{2}\left(\mathbb{R}^{d}\right)$ lies dense in $\mathcal{D}(\mathcal{A}) \cap C_{0}\left(\mathbb{R}^{d}\right)$. We now use a recursive argument. First, (B.1) implies that $C_{c}^{2}\left(\mathbb{R}^{d}\right) \subset R_{\lambda}^{Q}\left(C_{0}\left(\mathbb{R}^{d}\right)\right)$, hence $C_{0}\left(\mathbb{R}^{d}\right)$ lies in the closure $\operatorname{cl}\left(R_{\lambda}^{Q}\left(C_{0}\left(\mathbb{R}^{d}\right)\right)\right.$ of $R_{\lambda}^{Q}\left(C_{0}\left(\mathbb{R}^{d}\right)\right)$. But $R_{\lambda}^{Q}\left(\operatorname{cl}\left(R_{\lambda}^{Q}\left(C_{0}\left(\mathbb{R}^{d}\right)\right)\right)\right) \subset \mathcal{D}(\mathcal{G})$ by the regularizing properties of the resolvent (see Dynkin (1965) [Chapter I.2]). Using (B.1) again, we conclude that $C_{c}^{2}\left(\mathbb{R}^{d}\right) \subset \mathcal{D}(\mathcal{G})$. Moreover, by writing $f=R_{\lambda}^{P} g$, we have that $(\lambda-\mathcal{G}) f=(\lambda-\mathcal{A}) f+\mathcal{R} f$ for all $f \in C_{c}^{2}\left(\mathbb{R}^{d}\right)$, whence $\mathcal{G}=\mathcal{A}-\mathcal{R}$ on $C_{c}^{2}\left(\mathbb{R}^{d}\right)$.

By the closedness of $\mathcal{G}$ it is immediate that $\Theta \subset \mathcal{D}(\mathcal{G})$ (see (A.2)). In particular, for $f_{u, V} \in \Theta, Q_{t} f_{u, V}(x)$ is the unique solution to the initial value problem

$$
\partial_{t} g(t, x)=\mathcal{G} g(t, x), \quad g(0, x)=f_{u, V}(x) .
$$

By inspection it is clear that $\exp (\tilde{A}(t, u, V)+\langle\tilde{B}(t, u, V)\rangle+\langle\tilde{C}(t, u, V) x, x\rangle)$ is this solution, where $\tilde{A}, \tilde{B}$ and $\tilde{C}$ are the solutions of the Riccati equations (3.4)-(3.6) with $F, R, T$ replaced by $\tilde{F}:=F-R_{0}, \tilde{R}:=R-R_{1}$ and $\tilde{T}:=T-R_{2}$, respectively. Notice here that $\left(\gamma+R_{0}, \delta+R_{1}, \Phi+R_{2}\right) \in \mathcal{E}$, since $\mathcal{E}$ is a convex cone. By the differentiability and continuity of $\tilde{A}, \tilde{B}$ and $\tilde{C}$ on $\mathbb{R}_{+} \times \mathcal{B}$ it follows that the quadratic property

$$
Q_{t} f_{u, V}(x)=\exp (\tilde{A}(t, u, V)+\langle\tilde{B}(t, u, V)\rangle+\langle\tilde{C}(t, u, V) x, x\rangle)
$$

holds for all $(t, u, V) \in \mathbb{R}_{+} \times \mathcal{B}$ and that $\left(Q_{t}\right)$ is regular, which proves the sufficiency part of the lemma.

For the other direction, suppose now that $\left(Q_{t}\right)$ is a regular quadratic semigroup with parameters $(\alpha, \beta, b, \tilde{\gamma}, \tilde{\delta}, \tilde{\Phi})$. With similar arguments as above, one first shows that

$$
R_{\lambda}^{Q} g=R_{\lambda}^{P}\left(g-\mathcal{R}\left(R_{\lambda}^{Q} g\right)\right)
$$

for all $g \in C_{0}\left(\mathbb{R}^{d}\right)$ with $R_{\lambda}^{Q} g \in \mathcal{D}(\mathcal{R})$. From this we deduce, as above, that $\Theta \subset$ $\mathcal{D}(\mathcal{A})$ and $\mathcal{A}=\mathcal{G}+\mathcal{R}$ on $\Theta($ see $(\mathrm{A} .2))$.

Now we look at the Riccati equations (3.4)-(3.6) with coefficients $F, R$ and $T$ determined by $\left(\alpha, \beta, b, \gamma:=\tilde{\gamma}-R_{0}, \delta:=\tilde{\delta}-R_{1}, \Phi:=\tilde{\Phi}-R_{2}\right)$. However, in contrast to the above situation, here it is not clear yet whether these parameters are admissible, that is, whether $(\gamma, \delta, \Phi)$ belongs to $\mathcal{E}$ or not. However, the positive maximum principle (see Ethier and Kurtz (1986) [Theorem 4.2.2]) states that, for every continuous function $f \in \mathcal{D}(\mathcal{A})$ such that $P_{t} f$ is continuous, we have $\mathcal{A} f\left(x_{0}\right) \leq$ 0 whenever $\sup _{x \in \mathbb{R}^{d}} f(x)=f\left(x_{0}\right)$. Apply this to $f_{\epsilon}:=f_{\epsilon u, \epsilon V}-f_{2 \epsilon u, 2 \epsilon V}, \epsilon>0$, and 
let $\epsilon$ tend to zero to see that necessarily $(\gamma, \delta, \Phi) \in \mathcal{E}$. We conclude, as above, that $\left(P_{t}\right)$ is a regular quadratic semigroup with the claimed parameters.

\section{REFERENCES}

[1] Ahn, D. H., R. F. Dittmar, and A. R. Gallant (2002): Quadratic Term Structure Models: Theory and Evidence. The Review of Financial Studies 15, 243-288.

[2] Boyle, P., And W. Tian (1999): Quadratic Interest Rate Models as Approximations to Effective Rate Models. Journal of Fixed Income 9, 69-81.

[3] Chen, L., And H. V. Poor (2002): Parametric Estimation of Quadratic Models for the Term Structure of Interest Rate. Working paper, Princeton University.

[4] Cheng, P., and O. Scaillet (2002): Linear-Quadratic Jump-Diffusion Modeling with Application to Stochastic Volatility, Working paper, University of Geneva.

[5] Cheridito, P., And D. Filipović (2003): Equivalent and Absolutely Continuous Measure Changes for Jump-Diffusion Processes, Working paper, Princeton University.

[6] Duffie, D., D. Filipović, and W. Schachermayer (2003): Affine Processes and Applications in Finance. The Annals of Applied Probability 13, 984-1053.

[7] Duffie, D., J. Pan, And K. J. Singleton (2000): Transform Analysis and Asset Pricing for Affine Jump-Diffusions. Econometrica 68, 1343-1376.

[8] Duffie, D., And K. J. Singleton (1999): Modeling Term Structures of the Defaultable Bonds. The Review of Financial Studies 12, 687-720.

[9] Dynkin, E. B. (1965): Markov Processes Vol.1. Springer-Verlag.

[10] Ethier, S. N., AND T. G. Kurtz (1986): Markov Processes: Characterization and Convergence, John Wiley \& Sons Inc.

[11] Freiling, G. (2002): A Survey of Nonsymmetric Riccati Equations. Linear Algebra and its Applications 351-352, 243-270.

[12] Gombani, A., and W. J. Runggaldier (2001): A Filtering Approach to Pricing in Multifactor Term Structure Models. International Journal of Theoretical and Applied Finance 4, 303-320.

[13] Heston, S. (1993): A Closed-Form Solution of Options with Stochastic Volatility with Applications to Bond and Currency Options. The Review of Financial Studies 6, 327-343.

[14] Filipović, D. (2001): A General Characterization of One Factor Affine Term Structure Models. Finance and Stochastics 3, 389-412.

[15] Filipović, D. (2002): Separable Term Structures and the Maximal Degree Problem. Mathematical Finance 12, 341-349.

[16] Jarrow, R. A., D. Lando, and S. A. Turnbull (1997): A Markov Model for the Term Structure of Credit Risk Spreads. The Review of Financial Studies 10, 481-523.

[17] Lando, D. (1998): On Cox Processes and Credit-Risky Securities. Review of Derivatives Research 1, 99-120.

[18] Leippold, M., ANd L. Wu (2002): Asset Pricing Under the Quadratic Class. Journal of Financial and Quantitative Analysis 37, 271-295.

[19] Leippold, M., And L. Wu (2003a): Design and Estimation of Quadratic Term Structure Models, European Finance Review 7, 47-73.

[20] Leippold, M., And L. Wu (2003b): Multi-Currency Quadratic Models: Theory and Evidence, Working paper, City University of New York.

[21] LevendorskiǏ, S. (2002): Pseudo-Diffusions and Quadratic Term Structure Models. Working paper, Rostov State University of Economics.

[22] Rogers, L. C. G., And D. Williams (1994): Diffusions, Markov Processes, and Martingales, Vol.1. Second Edition, John Wiley \& Sons Ltd., Chichester, UK. 
[23] Sato, K. (1999): Lévy Processes and Infinitely Divisible Distributions. Cambridge University Press, Cambridge, UK.

The address correspondence to Li Chen, Information Sciences and Systems, Princeton University, Princeton, NJ 08544, USA. The address Correspondence to Damir FiLipović, Federal Office of Private Insurance, Friedheimweg 14, CH-3003 Bern, SwitzerLand. The address correspondence to H. Vincent Poor, Information Sciences and Systems, Princeton University, Princeton, NJ 08544, USA.

E-mail address: $\{$ lichen, poor\}@princeton.edu; filipo@math.ethz.ch 